\title{
Respiratory syncytial virus-related hospitalization in premature infants without bronchopulmonary dysplasia: subgroup efficacy analysis of the IMpact-RSV trial by gestational age group
}

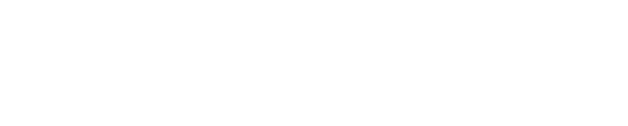

\section{Gerard Notario' \\ Pamela Vo ${ }^{2}$ \\ Katherine Gooch ${ }^{2}$ \\ Roger Deaton ${ }^{3}$ \\ Xionghua $\mathrm{Wu}^{4}$ \\ Brian Harris ${ }^{4}$ \\ Parthiv J Mahadevia ${ }^{4}$ \\ Pablo J Sánchez ${ }^{5}$ \\ 'Clinical Development and Medical Affairs, ${ }^{2}$ Global Heath Economics and Outcomes Research, ${ }^{3}$ Statistics, AbbVie Inc., North Chicago, IL, ${ }^{4}$ Medlmmune, LLC, Gaithersburg, MD, ${ }^{5}$ The Research Institute at Nationwide Children's Hospital, The Ohio State University, Columbus, $\mathrm{OH}$, USA}

Correspondence: Gerard Notario Clinical Development and Medical Affairs, AbbVie Inc., Dept R4M6, AP30-3 SW, I North Waukegan Road, North Chicago, IL 60064, USA

Tel + I 84793845 I 3

Fax + I 8479377775

Email gerard.notario@abbvie.com
Background: Respiratory syncytial virus (RSV) infections are common during childhood and are a major cause of hospitalization resulting from serious lower respiratory tract infections. Palivizumab is approved for prevention of serious lower respiratory tract disease caused by RSV infection in infants and young children at high risk of RSV disease. Although the previously published IMpact-RSV trial included the overall results for palivizumab prophylaxis in a cohort of premature infants who did not have bronchopulmonary dysplasia (BPD), there is an ongoing demand to understand the efficacy of palivizumab in premature infants without BPD by gestational age group.

Methods: This post hoc analysis assesses IMpact-RSV trial data within eleven gestational age groups for the cohort of 724 premature infants who were $\leq 6$ months of age and did not have BPD. RSV-related hospitalization rates along with corresponding 95\% exact binomial confidence intervals were determined for these specific gestational age groups. Relative reductions in risk of RSV-related hospitalizations for palivizumab versus placebo with corresponding $95 \%$ confidence intervals were also calculated.

Results: Analysis of a cohort of premature infants without BPD from the IMpact-RSV trial revealed that palivizumab consistently reduced RSV-related hospitalizations (64.5\%-100\%) versus placebo in all eleven gestational age groups evaluated. Palivizumab significantly decreased the relative risk of RSV-related hospitalization $(73 \%-82 \%, P<0.05)$ versus placebo in the $28-31$, 29-32, 29-33, 32-34, and 32-35 gestational week age groups. Relative reductions in risk for RSV-related hospitalizations were substantial for the moderate/late preterm groups $(82 \%$ for both the 32-34 and 32-35 gestational week age groups).

Conclusion: Palivizumab effectively reduced RSV-related hospitalization rates in premature infants without BPD across all gestational age groups evaluated. In particular, reductions in RSV-related hospitalizations were substantial for the moderate/late preterm groups (32-34 and 32-35 gestational weeks).

Keywords: respiratory syncytial virus, bronchopulmonary dysplasia, palivizumab, gestational age, prophylaxis

\section{Introduction}

Respiratory syncytial virus (RSV) is the most frequent cause of childhood lower respiratory tract infections (LRTI) worldwide and is a major cause of hospitalizations resulting from severe LRTI. ${ }^{1}$ In 2005, approximately 33 million RSV-related LRTI occurred worldwide in children under 5 years of age, and at least 3.4 million of these cases required hospitalization. ${ }^{1}$ 
In the randomized, placebo-controlled IMpact-RSV study, palivizumab prophylaxis significantly decreased the overall rate of RSV-related hospitalization by $55 \%$ among children with a history of preterm birth ( $\leq 6$ months of age) and/or bronchopulmonary dysplasia (BPD, $\leq 24$ months of age). ${ }^{2}$ Rates of adverse events were similar for palivizumabtreated and placebo-treated infants in the IMpact-RSV trial. Injection site reactions were uncommon, and few infants $(0.3 \%)$ had to discontinue injections because of palivizumabrelated adverse events. Palivizumab was subsequently approved by the US Food and Drug Administration for the prevention of serious LRTI due to RSV in pediatric patients at high risk of RSV disease. ${ }^{3}$

During the years since palivizumab was approved and as its use increased, payers and/or guideline authorities from around the world have initiated and are still revising clinical assessment criteria to determine which children might benefit most from palivizumab prophylaxis and where it is most costeffective. The criteria for eligibility include pediatric patients at high risk of RSV infection ${ }^{4}$ and vary from country to country. Because BPD has always been considered an important risk factor for RSV-related disease in children regardless of gestational age, these patients are typically recommended for prophylaxis. However, today there is an ongoing demand to understand the efficacy of palivizumab in different gestational age groups of premature infants without BPD.

Although the published IMpact-RSV trial included efficacy results for the cohort of premature infants without BPD, it did not report efficacy results by gestational age group. Thus, the objective of the present study was to perform post hoc analyses of the IMpact-RSV trial data to determine RSV-related hospitalization rates and relative risk reductions within gestational age groups of premature infants without BPD that are of particular interest and importance to payers and/or guideline authorities.

\section{Materials and methods Data source}

The IMpact-RSV trial was a multicenter, randomized (palivizumab [Synagis ${ }^{\circledR}$, MedImmune, LLC, Gaithersburg, MD, USA and AbbVie Inc., North Chicago, IL, USA] to placebo, 2:1), double-blind, placebo-controlled trial conducted during the 1996-1997 northern hemisphere RSV season at 139 centers in the USA, Canada, and UK. The trial was approved by the institutional review board for each center and parental consent was obtained for all infants enrolled in the trial. The IMpact-RSV trial was designed to evaluate the safety and efficacy of monthly prophylaxis with palivizumab in reducing the incidence of hospitalization due to RSV among high-risk infants. The results of the pivotal study have been published previously. ${ }^{2}$ In the present study, the post hoc analysis includes infants born at $\leq 35$ weeks' gestational age without BPD who were aged $\leq 6$ months ( 6 months +0 days) at the time of study entry.

Children were eligible to participate in the IMpact-RSV trial if they were $\leq 35$ weeks' gestational age and $\leq 6$ months' chronological age, or $\leq 24$ months' chronological age with a clinical diagnosis of BPD that required ongoing medical treatment within 6 months of enrollment. Gestational age was based on the medical record that described clinical assessment of the child at the time of study enrollment. Participants were to receive five monthly intramuscular injections of either palivizumab $(15 \mathrm{mg} / \mathrm{kg}$ ) or placebo (same formulation, except without antibody and with $0.02 \%$ Tween 80 added). RSV-related infections were identified by testing respiratory secretions for RSV antigen using commercially available tests in pediatric patients who were hospitalized with clinical signs and symptoms of LRTI.

\section{Analysis of data}

The baseline characteristics of the two treatment groups were compared using the two-sample $t$-test for age and weight, and Fisher's Exact test for other parameters. RSV-related hospitalization rates along with corresponding $95 \%$ exact binomial confidence intervals were determined within eleven premature gestational age groups. In addition, relative reductions in risk of RSV-related hospitalizations for palivizumab versus placebo were calculated, and the corresponding $95 \%$ confidence intervals were constructed using the exact conditional method on the total number of RSV-related hospitalizations with mid-probability adjustment. No adjustments for multiple comparisons were made. The following non-mutually exclusive gestational age groups were evaluated: $<28,<29$, 28-31, 29-30, 29-31, 29-32, 29-33, 32-34, 32-35, 33-34, and 33-35 weeks. Gestational age was described in terms of full weeks, where the interval for a week ranged from the full week number +0 days through the full week number +6 days. For example, the category 29-32 weeks included the interval week $29+0$ days (203 days) through week $32+6$ days (ie, 203-230 days). A total of 740 infants without BPD were included in the original analysis of the IMpact-RSV trial. ${ }^{2}$ However, 16 of these infants were $>6$ months in chronological age and were excluded from this analysis. Safety was not examined by gestational age group in this analysis or in the original trial. 
Table I Demographics and baseline characteristics by gestational age

\begin{tabular}{|c|c|c|c|c|}
\hline $\begin{array}{l}\text { Gestational } \\
\text { age (weeks) }\end{array}$ & Characteristics & $\begin{array}{l}\text { Placebo } \\
(n=230)\end{array}$ & $\begin{array}{l}\text { Palivizumab } \\
(n=494)\end{array}$ & $P$-value \\
\hline \multirow[t]{6}{*}{$<28$} & Number of infants & 17 & 53 & \\
\hline & Age at enrollment, mean (months) & 3.7 & 3.8 & 0.80 \\
\hline & Male, \% & 41.2 & 49.1 & 0.59 \\
\hline & White, \% & 52.9 & 54.7 & 1.00 \\
\hline & Weight at enrollment, mean $(\mathrm{kg})$ & 3.3 & 3.6 & 0.42 \\
\hline & Multiple birth, \% & 35.3 & 43.4 & 0.59 \\
\hline \multirow[t]{6}{*}{$<29$} & Number of infants & 40 & 102 & \\
\hline & Age at enrollment, mean (months) & 3.6 & 3.7 & 0.87 \\
\hline & Male, $\%$ & 50.0 & 52.9 & 0.85 \\
\hline & White, \% & 50.0 & 56.9 & 0.57 \\
\hline & Weight at enrollment, mean (kg) & 3.6 & 3.6 & 0.92 \\
\hline & Multiple birth, \% & 25.0 & 37.3 & 0.24 \\
\hline \multirow[t]{6}{*}{$28-31$} & Number of infants & 104 & 220 & \\
\hline & Age at enrollment, mean (months) & 3.2 & 3.2 & 0.89 \\
\hline & Male, \% & 55.8 & 50.0 & 0.34 \\
\hline & White, \% & 59.6 & 60.0 & 1.00 \\
\hline & Weight at enrollment, mean (kg) & 3.8 & 3.8 & 0.96 \\
\hline & Multiple birth, \% & 23.1 & 35.9 & 0.02 \\
\hline \multirow[t]{6}{*}{$29-30$} & Number of infants & 53 & 117 & \\
\hline & Age at enrollment, mean (months) & 3.2 & 3.3 & 0.56 \\
\hline & Male, \% & 54.7 & 51.3 & 0.74 \\
\hline & White, \% & 62.3 & 59.8 & 0.87 \\
\hline & Weight at enrollment, mean (kg) & 3.7 & 3.9 & 0.34 \\
\hline & Multiple birth, \% & 24.5 & 36.8 & 0.16 \\
\hline \multirow[t]{6}{*}{$29-31$} & Number of infants & 81 & $|7|$ & \\
\hline & Age at enrollment, mean (months) & 3.1 & 3.2 & 0.82 \\
\hline & Male, $\%$ & 55.6 & 48.0 & 0.28 \\
\hline & White, \% & 63.0 & 60.2 & 0.78 \\
\hline & Weight at enrollment, mean $(\mathrm{kg})$ & 3.8 & 3.9 & 0.86 \\
\hline & Multiple birth, \% & 24.7 & 37.4 & 0.046 \\
\hline \multirow[t]{6}{*}{$29-32$} & Number of infants & 117 & 256 & \\
\hline & Age at enrollment, mean (months) & 3.0 & 3.0 & 0.95 \\
\hline & Male, \% & 53.8 & 51.2 & 0.66 \\
\hline & White, \% & 64.1 & 61.3 & 0.65 \\
\hline & Weight at enrollment, mean $(\mathrm{kg})$ & 3.9 & 3.9 & 0.87 \\
\hline & Multiple birth, \% & 29.1 & 36.3 & 0.20 \\
\hline \multirow[t]{6}{*}{$29-33$} & Number of infants & 143 & 327 & \\
\hline & Age at enrollment, mean (months) & 2.9 & 3.0 & 0.55 \\
\hline & Male, \% & 51.0 & 52.6 & 0.76 \\
\hline & White, \% & 65.7 & 60.9 & 0.35 \\
\hline & Weight at enrollment, mean (kg) & 3.9 & 4.0 & 0.50 \\
\hline & Multiple birth, \% & 31.5 & 34.9 & 0.53 \\
\hline \multirow[t]{6}{*}{$32-34$} & Number of infants & 102 & 204 & \\
\hline & Age at enrollment, mean (months) & 2.6 & 2.7 & 0.57 \\
\hline & Male, \% & 51.0 & 57.4 & 0.33 \\
\hline & White, \% & 66.7 & 58.3 & 0.17 \\
\hline & Weight at enrollment, mean (kg) & 4.1 & 4.1 & 0.95 \\
\hline & Multiple birth, \% & 34.3 & 33.8 & 1.00 \\
\hline \multirow[t]{6}{*}{$32-35$} & Number of infants & 109 & 221 & \\
\hline & Age at enrollment, mean (months) & 2.7 & 2.8 & 0.60 \\
\hline & Male, \% & 52.3 & 57.5 & 0.41 \\
\hline & White, \% & 66.1 & 59.7 & 0.28 \\
\hline & Weight at enrollment, mean (kg) & 4.2 & 4.2 & 0.99 \\
\hline & Multiple birth, \% & 33.0 & 32.6 & 1.00 \\
\hline
\end{tabular}


Table I (Continued)

\begin{tabular}{lllll}
\hline $\begin{array}{l}\text { Gestational } \\
\text { age (weeks) }\end{array}$ & Characteristics & $\begin{array}{l}\text { Placebo } \\
(\mathbf{n = 2 3 0 )}\end{array}$ & $\begin{array}{l}\text { Palivizumab } \\
\text { (n=494) }\end{array}$ \\
\hline $33-34$ & Number of infants & 66 & 119 & $\begin{array}{c}\text { P-value } \\
\end{array}$ \\
& Age at enrollment, mean (months) & 2.5 & 2.7 & 0.45 \\
& Male, \% & 51.5 & 57.1 & 0.54 \\
& White, \% & 66.7 & 54.6 & 0.12 \\
& Weight at enrollment, mean $(\mathrm{kg})$ & 4.2 & 4.3 & 0.85 \\
$33-35$ & Multiple birth, \% & 31.8 & 33.6 & 0.87 \\
& Number of infants & 73 & 136 & 0.49 \\
& Age at enrollment, mean (months) & 2.6 & 2.8 & 0.66 \\
& Male, \% & 53.4 & 57.4 & 0.30 \\
& White, \% & 65.8 & 57.4 & 0.89 \\
\hline
\end{tabular}

\section{Results}

\section{Study population for subgroup analysis}

Data from 724 premature infants who were $\leq 6$ months of age and did not have BPD were evaluated (494 on palivizumab and 230 on placebo). To demonstrate that differences between prophylactic treatment groups were not confounded by differences in potential risk factors, baseline characteristics were compared across the gestational age groups (Table 1). The distributions of age, sex, race, and weight at enrollment were similar between palivizumab-treated and placebo-treated infants for each gestational age group. Across treatment groups, mean age at time of enrollment ranged from 2.5 to 3.7 months, mean weight at enrollment ranged from 3.3 to $4.3 \mathrm{~kg} ; 41 \%-58 \%$ of the infants were male and $50 \%-67 \%$ were of white race across all gestational age groups. Multiple birth rates were similar between palivizumab-treated and placebo-treated infants, except for the 28-31 and 29-31 gestational week age groups, where palivizumabtreated infants had a significantly higher multiple birth rate than placebo-treated infants (36\% versus $23 \%, P=0.02$, and $37 \%$ versus $25 \%, P=0.047$, respectively).

\section{Relative risk reductions of palivizumab versus placebo for RSV-related hospitalization}

Table 2 summarizes the incidence of RSV-related hospitalization, relative risk reductions versus placebo, and associated $95 \%$ confidence intervals by gestational age group for palivizumab-treated and placebo-treated infants. Palivizumab consistently reduced RSV-related hospitalizations $(64.5 \%-$ $100 \%$ ) versus placebo in premature infants without BPD in all gestational age groups evaluated. Relative reductions in risk for RSV-related hospitalizations were substantial for the moderate/late preterm groups (82\% for both the 32-34 and 32-35 gestational week age groups). Palivizumab decreased the relative risk of RSV-related hospitalization significantly $(P<0.05)$ versus placebo in the 28-31, 29-32, 29-33, 32-34, and $32-35$ gestational week age groups.

Table 2 Incidence of RSV-related hospitalization and relative risk reductions by gestational age groups in premature infants without bronchopulmonary dysplasia

\begin{tabular}{|c|c|c|c|c|c|c|c|c|}
\hline \multirow{2}{*}{$\begin{array}{l}\text { Gestational } \\
\text { age (weeks) }\end{array}$} & \multicolumn{3}{|c|}{ Placebo $(n=230)$} & \multicolumn{3}{|c|}{ Palivizumab $(n=494)$} & \multirow[t]{2}{*}{ RRR (\%) } & \multirow[t]{2}{*}{$95 \% \mathrm{Cl}^{*}$} \\
\hline & $n / N$ & $\%$ & $95 \% \mathrm{CI} *$ & $n / N$ & $\%$ & $95 \% \mathrm{Cl}^{*}$ & & \\
\hline$<28$ & $1 / 17$ & 5.9 & $0.1-28.7$ & $1 / 53$ & 1.9 & $0.0-10.1$ & 67.9 & $-969.4,99.0$ \\
\hline$<29$ & $4 / 40$ & 10.0 & $2.8-23.7$ & $2 / 102$ & 2.0 & $0.2-6.9$ & 80.4 & $-8.3,97.4$ \\
\hline $28-31^{\dagger}$ & $7 / 104$ & 6.7 & $2.7-13.4$ & $4 / 220$ & 1.8 & $0.5-4.6$ & 73.0 & $7.7-95.1$ \\
\hline $29-30$ & $1 / 53$ & 1.9 & $0.0-10.1$ & $0 / 117$ & 0.0 & $0.0-3.1$ & 100.0 & $-564.0,100.0$ \\
\hline $29-31$ & $4 / 81$ & 4.9 & I.4-12.2 & $3 /|7|$ & 1.8 & $0.4-5.0$ & 64.5 & $-64.0,95.4$ \\
\hline $29-32^{\dagger}$ & $9 / 117$ & 7.7 & $3.6-14.1$ & $4 / 256$ & 1.6 & $0.4-4.0$ & 79.7 & $35.7-96.9$ \\
\hline $29-33^{\dagger}$ & $13 / 143$ & 9.1 & $4.9-15.0$ & $6 / 327$ & 1.8 & $0.7-4.0$ & 79.8 & $49.0-94.2$ \\
\hline $32-34^{\dagger}$ & $11 / 102$ & 10.8 & $5.5-18.5$ & $4 / 204$ & 2.0 & $0.5-4.9$ & 81.8 & $45.4-96.5$ \\
\hline $32-35^{\dagger}$ & $11 / 109$ & 10.1 & $5.1-17.3$ & $4 / 221$ & 1.8 & $0.5-4.6$ & 82.1 & $45.9-96.6$ \\
\hline $33-34$ & $6 / 66$ & 9.1 & $3.4-18.7$ & $3 / 119$ & 2.5 & $0.5-7.2$ & 72.3 & $-\mid 4.1,96.3$ \\
\hline $33-35$ & $6 / 73$ & 8.2 & $3.1-17.0$ & $3 / 136$ & 2.2 & $0.5-6.3$ & 73.2 & $-10.8,96.4$ \\
\hline
\end{tabular}

Notes: *Exact binomial 95\% confidence interval; ’statistically significant difference between the palivizumab and placebo treatment groups $(P<0.05)$.

Abbreviations: $\mathrm{Cl}$, confidence interval; RRR, relative risk reduction of palivizumab versus placebo; RSV, respiratory syncytial virus. 


\section{Discussion}

Although guidelines exist that provide recommendations for the prophylaxis of RSV-related infections in major risk groups, ie, very early preterm infants ( $<29$ weeks' gestational age), those $\leq 35$ weeks' gestational age, and those with BPD, ${ }^{5-7}$ decisions regarding which infants should receive prophylaxis vary worldwide and are being made on the basis of individual circumstances. Thus, understanding the efficacy of palivizumab in different premature infant groups as observed within a randomized controlled trial will be an important part of the decision-making process. This post hoc subgroup analysis of the IMpact-RSV trial demonstrates the consistency of palivizumab (ranging from $64.5 \%$ to $100 \%$ ) in reducing RSV-related hospitalization in premature infants without BPD across eleven gestational age groups. To our knowledge, this is the first analysis that reports efficacy by gestational age group for premature infants without BPD.

It is noteworthy that a high degree of efficacy was achieved with palivizumab in later preterm infants ( $\geq 32$ weeks) as well as in very early preterm infants without BPD. High efficacy against RSV hospitalization ( $82 \%$ relative risk reduction) was also demonstrated in preterm infants of 33-35 weeks' gestational age in the recent randomized, blinded, placebo-controlled MAKI trial (Effect of palivizumab on respiratory syncytial virus-associated burden of disease) conducted in the Netherlands. ${ }^{8}$ Although the IMpact-RSV trial demonstrated an overall 55\% reduction versus placebo in RSV hospitalizations among palivizumab recipients, the trial enrolled a large number of infants with BPD. In the general population, the incidence of BPD among preterm infants is low and has decreased in recent years with improvements in medical care. Using contemporary estimates of the incidence of BPD and of prematurity without BPD, ${ }^{9,10}$ the weighted estimate of the efficacy of palivizumab among all preterm infants is approximately $75 \%$. This estimate was derived by summing the products of the condition-specific relative risk reductions observed in the IMpact-RSV trial and contemporary estimates of the relative frequency of each condition.

IMpact-RSV was a randomized, placebo-controlled trial powered to detect statistically significant differences in RSV hospitalization rates between palivizumab-treated and placebo-treated pediatric groups. Ninety-nine percent of the children in both groups completed the protocol, and 93\% received all five scheduled injections. ${ }^{2}$ This post hoc analysis assesses the IMpact-RSV trial data in the subgroup of premature infants without BPD for eleven gestational age groups which are of particular interest and importance to payers and guideline authorities. The results demonstrate that efficacy estimates for groups of premature infants treated with palivizumab were consistently high (ranging from $64.5 \%$ to $100 \%$ ). However, a statistical limitation due to the small sample sizes for some groups results in large confidence intervals around the efficacy point estimates, suggesting a need for cautious interpretation. Although the data are from the 1996-1997 trial, they still provide useful information regarding the efficacy of palivizumab, because this analysis helps to close the knowledge gap regarding the efficacy of palivizumab within specific premature gestational age groups without BPD. Furthermore, the recently published MAKI trial supports these findings, particularly in moderate/late preterm infants, given that it demonstrates an $82 \%$ relative risk reduction for palivizumab with regard to RSV hospitalization among infants of 33-35 weeks' gestational age. $^{8}$

\section{Conclusion}

The results of this analysis suggest that palivizumab effectively reduced RSV-related hospitalization rates in premature infants without BPD across all the gestational age groups evaluated. In particular, reductions in RSV-related hospitalizations were substantial (82\%) for the moderate/late preterm groups (32-34 and 32-35 weeks' gestational age).

\section{Author contributions}

All authors participated in the design, implementation, analysis, and interpretation of the study, writing of the manuscript, and the decision to submit for publication. All authors read and approved the final manuscript.

\section{Disclosure}

GN, PV, and KG are employees of AbbVie Inc., and may hold stocks or options; RD is a former employee of AbbVie Inc., and may hold stocks or options. BH and PJM are employees of MedImmune and may hold stocks or options; XW is a former employee of MedImmune and may hold stocks or options. PJS is a consultant for AbbVie Inc., and has received honoraria. This study was supported by AbbVie Inc. The study sponsor was involved in all stages of the study conduct and analysis, and in the development of the manuscript and its approval for submission. All authors had full access to the data and had final responsibility for the decision to submit for publication. Medical writing and editing services were provided by Joann Hettasch, of Arbor Communications, Inc., Ann Arbor, MI, USA. The manuscript preparation was funded by AbbVie Inc., which also paid the processing charge for this paper. 


\section{References}

1. Nair H, Nokes DJ, Gessnere BD, et al. Global burden of acute lower respiratory infections due to respiratory syncytial virus in young children: a systematic review and meta-analysis. Lancet. 2010;375: $1545-1555$.

2. IMpact-RSV Study Group. Palivizumab, a humanized respiratory syncytial virus monoclonal antibody, reduced hospitalization from respiratory syncytial virus infection in high risk infants. Pediatrics. 1998;102:531-537.

3. Synagis (Prescribing information). Gaithersburg, MD, USA: MedImmune; 2013.

4. Langley GF, Anderson LJ. Epidemiology and prevention of respiratory syncytial virus infections among infants and young children. Pediatr Infect Dis J. 2011;30:510-517.

5. American Academy of Pediatrics. Respiratory syncytial virus. In: Pickering K, editor. Red Book: 2012 Report of the Committee on Infectious Diseases. 29th ed. Elk Grove Village, IL, USA: American Academy of Pediatrics; 2012.
6. Joint Committee on Vaccination and Immunisation. Statement on Immunisation for Respiratory Syncytial Virus. Available from: http:// webarchive.nationalarchives.gov.uk/20130107105354/http://www. dh.gov.uk/prod_consum_dh/groups/dh_digitalassets/@dh/@ab/ documents/digitalasset/dh_120395.pdf. Accessed March 16, 2014.

7. Robinson JL; Canadian Paediatric Society, Infectious Diseases and Immunization Committee. Position Statement. Preventing respiratory syncytial virus infections. Paediatr Child Health. 2011;16:488-490.

8. Blanken MO, Rovers MM, Molenaar JM, et al. Respiratory syncytial virus and recurrent wheeze in healthy preterm infants. $N$ Engl J Med. 2013;368:1791-1799.

9. Escobar GJ, Masaquel AS, Li SX, Walsh EM, Kipnis P. Persistent recurring wheezing in the fifth year of life after laboratory-confirmed, medically attended respiratory syncytial virus infection in infancy. $B M C$ Pediatr. 2013;13:97.

10. Yoder BA, Harrison M, Clark RH. Time-related changes in steroid use and bronchopulmonary dysplasia in preterm infants. Pediatrics. 2009;124:673-679.

\section{Publish your work in this journal}

Pediatric Health, Medicine and Therapeutics is an international, peerreviewed, open access journal publishing original research, reports, editorials, reviews and commentaries. All aspects of health maintenance, preventative measures and disease treatment interventions are addressed within the journal. Practitioners from all disciplines are invited to submit their work as well as healthcare researchers and patient support groups. The manuscript management system is completely online and includes a very quick and fair peer-review system. Visit http://www.dovepress.com/ testimonials.php to read real quotes from published authors.

Submit your manuscript here: http://www.dovepress.com/pediatric-health-medicine-and-therapeutics-journal 